\title{
RELAÇÃO DO COMPORTAMENTO ALIMENTAR E O ESTADO NUTRICIONAL DE ESCOLARES DAS REDES DE ENSINO FUNDAMENTAL DAS REDES PÚBLICAS E PRIVADAS - REVISÃO INTEGRATIVA
}

\author{
RELATIONSHIP OF FOOD BEHAVIOR AND THE NUTRITIONAL \\ STATE OF SCHOOLS IN PUBLIC AND PRIVATE EDUCATION \\ NETWORKS - INTEGRATIVE REVIEW
}

\author{
Andrezza Oliveira Barros ${ }^{1}$ \\ Andréia Oliveira Barros Sousa ${ }^{2}$ \\ Danielle Figueiredo Patrício ${ }^{3}$ \\ Rafaela Ramos Dantas ${ }^{4}$
}

RESUMO: Objetivo: Relacionar o comportamento alimentar com estado nutricional de escolares do ensino fundamental de redes públicas e privadas. Método: Trata-se de uma pesquisa exploratório, descritivo, do tipo revisão integrativa, com abordagem qualitativa, acerca do estado nutricional e comportamento alimentar dos escolares do ensino fundamental no país, desenvolvido através de pesquisas realizadas de estudos produzidos sobre a temática de acordo com os descritores. Resultados: Observou-se um elevado número de escolares com excesso de peso em escolas pública e privadas, com uma prevalência maior nas redes particulares. Das escolas estudas apenas as de ensino pública possuía um programa de educação alimentar implantado. Todas as crianças classificadas com excesso de peso possuíam hábitos alimentares não-saudáveis. Conclusão: Foi possível analisar nesse estudo que existe uma intrínseca relação entre a obesidade, comportamento alimentar e o ambiente no qual o indivíduo encontra-se inserido. Necessário se faz buscar estratégias alimentares mais saudáveis para que as escolas deixem de ser ambientes obesogênicos e se tornem referência não só na educação, mas na saúde.

Descritores: Escola. Nutrição saudável. Obesidade infantil.

\footnotetext{
${ }^{1}$ Especialista em Urgência, Emergência e UTI, Graduada pela Faculdade de Ciências Médicas - FCM e Docente do Departamento de Enfermagem da UEPB. Email:dezabarros@gmail.com.

${ }^{2}$ Doutoranda em pela USP, Docente do Departamento de Enfermagem da UFCG.

3 Mestre em Psicologia da Saúde e Graduada pela Universidade Estadual da Paraíba - UEPB, Docente do Departamento de Enfermagem da UEPB.

${ }^{4}$ Mestre em Saúde da Criança e Adolescente pela Universidade Federal de Pernambuco - UFPE, Docente do Departamento de Enfermagem da UEPB.
} 
ABSTRACT: Objective: Relate eating behavior with nutritional status of elementary school students from public and private schools. Method: This is an exploratory, descriptive, integrative review type study, with a qualitative approach, about the nutritional status and eating behavior of elementary school students in the country, developed through research carried out on studies produced on the theme according to the descriptors. Results: There was a high number of overweight students in public and private schools, with a higher prevalence in private schools. Of the schools studied, only those in public education had a food education program in place. All children classified as overweight had unhealthy eating habits. Conclusion: It was possible to analyze in this study that there is an intrinsic relationship between obesity, eating behavior and the environment in which the individual is inserted. It is necessary to seek healthier eating strategies so that schools cease to be obesogenic environments and become a reference not only in education, but in health.

Descriptors: School. Healthy nutrition. Childhood obesity. 


\section{INTRODUÇÃO}

A obesidade é uma doença crônica, multifatorial definida como alto acúmulo de gordura no corpo quando comparado à massa magra. Este aumento do tecido adiposo está relacionado com aspectos comportamentais, nutricionais, condições sociais e estilo de vida. Segundo a Organização Mundial de Saúde em 2016 mundialmente cerca de 1,9 milhões de adultos acima de 18 anos estavam sobre peso e destes $13 \%$ eram obesos. Essa doença atualmente é considerada um problema de saúde pública presente não só em adultos, mas também em crianças e adolescente (WHO, 2016).

Em 2022 haverá uma maior prevalência de criança e adolescente (5 - 19 anos) obesos em relação à epidemiologia de desnutridos. Esse crescimento é preocupante, pois esse fator compromete intimamente a saúde dessas crianças e adolescentes. Esses dados são mundiais, mas existe uma predominância em países de baixa e média renda (EZZATI, 2017).

No Brasil, segundo dados do IBGE (2015), na faixa etária de 13 a 17 anos $15,9 \%$ estavam sobrepeso e desses $7,8 \%$ apresentavam obesidade. No nordeste nessa mesma faixa etária 14, 1 \% pelo índice de massa corpórea (IMC) eram sobrepeso e desses $6,4 \%$ obesos.

A obesidade infantil se tornou uma epidemia e a grande preocupação é que se tornou um fator de risco para desenvolvimento de doenças crônicas na vida adulta de forma precoce (LOPES, et. al. 2019).

As morbidades que a acompanham é o que mais preocupa, dentre elas podemos citar as síndromes metabólicas, como diabetes tipo 2, problemas cardiovasculares, dislipidemia, hipertensão arterial sistêmica, doenças hepáticas, distúrbios do sono, problemas ortopédicos, algumas formas de câncer e transtornos psicológico como ansiedade e depressão.(HOCKENBERRY, 2014). 
O mesmo autor destaca que a obesidade infantil tem relação com alguns fatores predisponente como genética, fatores hipotalâmicos, metabólicos, sociais, culturais e psicológicos.

O desenvolvimento da obesidade possui uma forte relação com um comportamento alimentar inadequado e redução da prática de atividades físicas. $\mathrm{E}$ nesse contexto é importante enfatizar que as atitudes parentais e um ambiente obesogênico possuem forte influência nestes dois aspectos citados (DANTAS; SILVA, 2019).

A identificação dos fatores de riscos é crucial para entender o processo de desenvolvimento da obesidade, e dessa maneira pode-se definir intervenções adequadas tanto para prevenção quanto para o tratamento da doença. Uma criança ou adolescente obeso poderá se tornar um adulto obeso e esse fator interferirá na saúde, qualidade e expectativa de vida desse indivíduo (BRASIL, 2017).

$\mathrm{Na}$ infância os pais ou cuidadores exercem forte influência em todos os aspectos de vida. As atitudes e práticas educacionais parentais serão determinantes importantes da personificação da criança e comportamento que influenciem na saúde, como hábitos alimentares e prática de exercício físico (SILVA E TEIXEIRA, 2018).

O comportamento alimentar na infância se inicia no contexto familiar sendo posteriormente moldada por aspectos psicológicos, econômicos, culturais e sociais. E estas influências, das atitudes parentais, estão relacionadas ao excesso de peso e obesidade infantil (DANTAS E SILVA, 2019).

O ambiente onde as crianças e os adolescentes estão inseridos, tem uma íntima relação com o surgimento da obesidade. Um ambiente obesogênico oferecerá oportunidades de escolhas de todos os aspectos induzindo o indivíduo a um comportamento alimentar inadequado e diminuição de práticas de atividades físicas (PEREIRA; BARROS; HEMAIS, 2019).

Os autores supracitados enfatizam que apesar da escola atuar na educação do ser humano, influenciando na personalidade e vários aspectos comportamentais, ela poderá se tornar também um ambiente obesogênico.

É possível observar que em muitas instituições educacionais os alimentos consumidos tanto por crianças e adolescentes não são consideráveis saudáveis, e 
que por serem muito atraentes acabam estimulando um consumo excessivo, propiciando consequentemente hábitos alimentares inadequados (PEREIRA; BARROS; HEMAIS, 2019).

O Programa Nacional de Alimentação Escolar (PNAE), foi criada pela Lei $n^{\circ}$ $11.947 / 2009$, com o intuito de melhorar o estado nutricional dos alunos matriculados em escolas públicas durante o período letivo, através do emprego de uma alimentação saudável e adequada que possam suprir todas as necessidades nutricionais, favorecendo um bom crescimento e desenvolvimento da criança e do adolescente para assim melhorar o rendimento escolar. Além disso uma de suas diretrizes está relacionada a educação alimentar para atuar na formação de hábitos alimentares saudáveis e prevenção de Doenças Crônicas não Transmissíveis (DCNT) (BRASIL, 2009).

Por isso investigar essa temática se torna relevante quando se compreende que as práticas parentais associadas a um ambiente obesogênico contribuem para o aumento da incidência da obesidade infanto-juvenil.

A partir desse questionamento pretende-se de forma geral identificar na literatura o estado nutricional e comportamento alimentar dos escolares do ensino fundamental no país, buscando descrever a relevância dessa instituição na mudança de uma postura obesogênica.

\section{MÉTODOS}

Trata-se de uma pesquisa exploratório, descritivo, do tipo revisão integrativa, com abordagem qualitativa, que segundo Botelho, Cunha, Macedo (2011) é um método específico, no qual sintetiza conhecimentos relevantes do passado da literatura para fornecer uma maior clareza de um fenômeno particular, objetivando analisar estudos anteriores sobre determinado tema e assim trazer melhorias para prática clínica.

O levantamento dos dados ocorreu em janeiro de 2020, sendo a fonte da coleta realizada em artigos científicos publicados em periódicos indexados nas 
bases de dados eletrônicas como Sistema Online de Busca e Análise de Literatura Médica (MEDLINE), Base de Dados de Enfermagem (BDENF) e Literatura Latino Americana e do Caribe em Ciências de Saúde (LILACS) e Caribe de Informação em Ciências da Saúde (BIREME). Para busca dos artigos foram utilizados os seguintes descritores: obesidade infantil AND escola AND nutrição saudável.

Para levantamento dos artigos a serem utilizados na pesquisa foram definidos como critérios de inclusão, estudos disponíveis na integra, no idioma português, publicados entre os anos de 2015 a 2020 e que respondessem a questão norteadora: estado nutricional e comportamento alimentar dos escolares do ensino fundamental no país. Foram excluídos artigos cujo conteúdo não estava de acordo com o objetivo proposto pela pesquisa.

Após análise inicial, realizou-se a seleção de 10 artigos das bases de dados designadas, sendo incluídos para leitura na integra 4 artigos, por atenderem os critérios de inclusão apresentados.

Os dados foram coletados após inclusão de palavras-chave na barra de pesquisa das bases de dados acima descrita, após isso procedeu-se a aplicação dos filtros que garantiram a coleta a partir dos critérios de inclusão e exclusão da pesquisa. Para melhor avaliação dos artigos foi realizada leitura prévia dos resumos para verificar real adequação aos objetivos desta pesquisa. Foi utilizado, para uma melhor compreensão das informações, um roteiro estruturado com as seguintes variáveis: nome do(s) autor(es), título do artigo, periódico indexado, ano, objetivos, principais resultados e considerações. Para uma melhor compreensão dos resultados, os artigos foram classificados sequencialmente de A1 a A4.

Para a análise dos dados utilizou-se a técnica da descrição analítica. Após a seleção dos artigos científicos ocorreu a leitura criteriosa dos textos e análise dos resultados das pesquisas. Em seguida, os trabalhos foram analisados e reduzidos a fichas com conteúdo relevante para esta pesquisa.

Durante todas as etapas analíticas foram confeccionados fichamentos para uma melhor organização e compreensão dos resultados, sendo estes categorizados baseando-se no método de revisão integrativa.

Em virtude da natureza científica da pesquisa, foram considerados os aspectos éticos presentes no capítulo III do Código de Ética dos Profissionais de 
Enfermagem. Sendo respeitado o princípio de autoria dos dados, através da utilização das normas de citações e referências elaboradas pela Associação Brasileira de Normas Técnicas (ABNT). Após a realização do estudo o seu resultado será publicado para o meio acadêmico e para a população a quem interessar objetivando o retorno social da pesquisa.

\section{RESULTADOS E DISCUSSÕES}

Ao analisar os artigos é notória a abordagem realizada sobre hábitos alimentares saudáveis dentro das escolas de todos os autores, e três destes estudos relacionam uma nutrição inadequada com o excesso de peso e obesidade em crianças.

Hábitos alimentares saudáveis ou não saudáveis da criança refletem na saúde na vida adulta. Alimentos apropriados ofertam todos os nutrientes adequados para esta população, promovendo um crescimento e desenvolvimento satisfatório. Além disso previne problemas á saúde e doenças crônicas não-transmissíveis ao longo prazo (ALVES, et. al., 2018).

Todos os estudos abordaram crianças, com idade que varia de 02 à 12 anos, matriculadas no ensino fundamental tanto de escolas públicas como privadas. Desses um abordou os dois simultaneamente, dois escolheram pesquisar apenas escola privada e uma apenas instituições de ensino público.

Os hábitos alimentares são moldados na infância, e seu comportamento tem influência nas escolhas dos alimentos na fase adulta. Muitos fatores estarão relacionados com a formação das práticas saudáveis em relação às escolhas dos alimentos das crianças, como por exemplo família, escola e mídias. Por este motivo é importante que a educação alimentar seja iniciada neste período, com a participação ativa das famílias juntamente com as escolas, para que juntas possam construir um comportamento alimentar adequado e saudável para esta criança e consequente prevenir as DCNT quando adulto (COSTA; RIBEIRO; SANTOS, 2019). 
Uma das pesquisas ressaltou o consumo de sobremesa oferecida pela escola privada. A oferta de frutas como sobremesas foi adequada e estava presente em grande parte dos dias da semana, mas sua aceitação foi baixa quando comparada ao consumo de doces, mesmo a oferta sendo menor que a de frutas (KREY et. al., 2018).

Tanto as instituições públicas e privadas possuem papel fundamental na formação de uma educação nutricional e alimentar na infância. Os estudos realizados na rede de ensino privada não mostraram nenhum programa voltado à educação alimentar e nutricional das crianças dentro das escolas privadas. Através dos resultados encontrados em suas pesquisas enfatizam a necessidade da inclusão dessas práticas educativas focada em uma alimentação saudável para promoção da redução do excesso de peso e da saúde desses estudantes (KREY et. al, 2018; PASSOS et. al., 2015).

Nas escolas públicas existe o Programa Nacional de Alimentação Escolar (PNAE), que além de permitir uma oferta nutricional adequada que favorece o crescimento e desenvolvimento da criança, proporciona ao mesmo tempo uma educação alimentar e nutricional para formação de um comportamento adequado em relação à alimentação e nutrição (BRASIL, 2009).

A obesidade infantil está intimamente ligada ao comportamento alimentar inadequado. Em um dos estudos analisados foi avaliado este comportamento, e observou-se que as crianças com excesso de peso possuem maior interesse e prazer pela comida, além de destacar a relação do aumento da ingesta de alimentos com o estado emocional. Essas crianças possuem dificuldades em regular o consumo de alimentos resultado no aumento de peso inadequado para idade (PASSOS et. al., 2015).

Em relação ao estado nutricional das crianças analisadas, observou-se um elevado número de escolares com excesso de peso em três das pesquisas tanto em escolas públicas quanto privadas.

Em relação a isso, o estudo de Grillo e seus colaboradores (2016) que buscou avaliar o estado nutricional de crianças do ensino público que tem implantado o PNAE, evidenciou uma prevalência alta de excesso de peso nesta população e 
todos estes possuíam risco cardiovascular quando aferido a circunferência abdominal.

Além de identificar o estado nutricional desses escolares, foi desenvolvido junto com a comunidade escolar um processo de educação nutricional através de oficinas e foi percebida uma educação efetiva com alto nível de satisfação de todos os participantes. Neste sentindo Grillo et. al (2016), destaca a importância da adesão de atividades educativas para impactar positivamente nos hábitos alimentares desses estudantes.

No estudo comparativo abordado por Krey et. al (2018), Paiva et.al.(2018) e Passos et. al (2015) foi constatado uma maior incidência de excesso de peso em crianças das escolas privadas comparadas as da rede pública de ensino da presente pesquisa. Paiva et. al (2018), acreditam que esta estatística tem relação com a ingesta de alimentos mais calóricos e com baixo teor nutritivo oferecidos pelos seus progenitores, pelo fato de serem alimentos práticos, rápidos e de fácil acesso. Além disso, não existe um programa de educação alimentar dentro das escolas particulares, observadas nestes estudos, que possam contribuir para promoção de hábitos alimentares saudáveis.

Dos artigos que abordaram o estado nutricional das crianças, foram utilizados a medida de IMC (Índice de Massa Corpórea) para avaliação e classificação da criança de acordo com o seu peso. Desses pode-se concluir uma alta prevalência de estudantes com excesso de peso (PASSOS et. al., 2015; GRILLO et. al. 2016; PAIVA, 2018 ).

Grillo et. al. (2016), consideraram outro dado antropométrico, a circunferência abdominal, para relação do excesso de peso com o risco de doenças cardiovasculares. Neste contexto, todos os escolares com IMC acima do normal, possuíam um acúmulo de gordura na região abdominal.

Outros dados foram abordados por Paiva et. al (2018), além do peso e altura, ele incluiu pressão arterial e alguns exames laboratoriais para traças o perfil lipídicos e glicemia. Neste aspectos constatou-se um elevado índice de dislipidemias e aumento da glicemia em um grande número das crianças classificadas com sobrepeso e obesidade. Não se obteve variação dos níveis pressóricos nesta população, o que pode estar relacionado à prática de exercício dentro da escola ou 
pela característica de cronicidade da hipertensão arterial que geralmente é lenta, podendo levar anos para sua manifestação (PAIVA et. al, 2018).

\section{CONCLUSÃO}

Foi possível analisar nesse estudo que existe uma intrínseca relação entre a obesidade, comportamento alimentar e o ambiente no qual o indivíduo encontra-se inserido. Em todos os artigos analisados percebeu-se que apesar de uma política nacional que se encontra implantada em todo o país, muitas instituições ainda não buscaram dentro dos seus muros efetivar e implementar a mesma.

Como consequência disso a cada dia que passa é constatado um aumento na incidência da obesidade infanto-juvenil, com uma perspectiva de um aumento de doenças que possuem relação direta com esse distúrbio.

É notório a reflexão da necessidade de se investir em campanhas e programas que direcionem as instituições escolares em estimular dietas mais saudáveis para crianças e adolescentes.

Esse trabalho precisa ser iniciado o mais precocemente possível, incluindo não só os jovens mais as famílias.

Necessário se faz buscar estratégias alimentares mais saudáveis para que as escolas deixem de ser ambientes obesogênicos e se tornem referência não só na educação, mas na saúde. 


\section{REFERÊNCIAS BIBLIOGRÁFICAS}

ALVES, N. I. et. al. Estado nutricional de crianças em creches de Carapicuíba-SP comparado ao de seus pais. Reciis - Rev Eletron Comun Inf Inov Saúde. 2018 jul.set.;12(3):310-22. Disponível em: https://www.reciis.icict.fiocruz.br/index.php/reciis/article/view/1371/2225. Acesso em 06 de janeiro de 2020.

BARDIN, L. Análise de Conteúdo. Rio de Janeiro: Edições 70 Brasil, 2011.

BRASIL. Manual de diretrizes para o enfrentamento da obesidade na saúde suplementar brasileira. [recurso eletrônico]. Rio de Janeiro: ANS, 2017. Disponível em: http://www.ans.gov.br/images/total_obesidade_10_04_18.pdf. Acesso em 06 de janeiro de 2020.

Brasil. Lei $n^{0} 11.947$ de 16 de julho de 2009. Dispõe sobre o atendimento da alimentação escolar e do Programa Dinheiro Direto na Escola aos alunos da educação básica. Diário Oficial da União 2009; 16 jun. Disponível em: www.sed.sc.gov.br > 2344-lei-n-11-947-2009-pnae4129 Acesso em 06 de janeiro de 2020.

COSTA, R. A. O.S; RIBEIRO, J. L. O. A; SANTOS, M. R. A contribuição da educação infantil para a formação de bons hábitos alimentares na criança de 0 a 6 anos. Revista Eletrônica de Ciências da Saúde - UNIPLAN. Águas Claras/DF, V1,

N. 1, 2019. Disponível em: http://www.revistauniplan.com.br/index.php/REVHUMANAS/article/view/27/19. Acesso em 06 de janeiro de 2020.

DANTAS, R. R.; SILVA, G. A. P. O papel do ambiente obesogênico e dos estilos de vida parentais no comportamento alimentar infantil. São Paulo: Revista Paulista de Pediatria, 2019. Disponivel em: http://www.scielo.br/scielo.php?script=sci_arttext\&pid=S0103$05822019005009101 \&$ Ing=en\&tIng=en. Acesso em 06 de janeiro de 2020.

EZZATI, M. Worldwide trends in body-mass index, underweight, overweight, and obesity from 1975 to 2016: a pooled analysis of 2416 population-based measurement studies in 128.9 million children, adolescents, and adults. The lancet, London. Vol 390. P. 2607, 2017. Disponivel em: https://www.thelancet.com/action/showPdf?pii=S0140-6736\%2817\%2932129-3 . Acesso em 06 de janeiro de 2020.

GRILLO, L. P. et. al. Estado nutricional e práticas de educação nutricional em escolares. O Mundo da Saúde, São Paulo; v.40Ezzati, M. 2017, p. 230-238. 2016. Disponível em: http://bvsms.saude.gov.br/bvs/periodicos/mundo_saude_artigos/estado_nutricional.pdf Acesso em 06 de janeiro de 2020.

Hockenberry, M. J.; Wilson, D. Wong. Fundamentos de Enfermagem Pediátrica. Rio de Janeiro: Elsevier, $9^{\mathrm{a}}$ ed, 2014.

IBGE. Pesquisa Nacional de Saúde do Escolar : Percentual de escolares com idade de 13 a 17 anos, distribuído pelo estado nutricional, por sexo e grupo de idade do escolar no Brasil. 2015. Disponível em: https://sidra.ibge.gov.br/tabela/6366. Acesso em 06 de janeiro de 2020.

KREY, I. P. et. al. Consumo de sobremesas por alunos e destino das sobras em escola de educação infantil e fundamental privada da cidade de São Paulo. Higiene Alimentar - Vol.32 - $n^{\circ} 282 / 283 \quad$ - Julho/Agosto de 2018. Disponível em: https://pdfs.semanticscholar.org/5dc3/9171eda0a20dcdcb8f587681a627eff13a4d.pdf?_ga=2.175 
187874.1629790374.1580329999-1226020820.1579475117. Acesso em 06 de janeiro de 2020.

Lopes, C. A. O. et. al. Prevenção da obesidade infantil: uma proposta educativa. Revista interfaces: Minas Gerais, v.7, n.1, 2019. Disponível em: https://www.ufmg.br/proex/revistainterfaces/index.php/IREXT/article/view/285. Acesso em 06 de janeiro de 2020.

PAIVA A. C. T. et. al . Obesidade Infantil: análises antropométricas, bioquímicas, alimentares e estilo de vida. Rev Cuid. v.9, i.3.575. 2018. Disponível em http://www.scielo.org.co/pdf/cuid/v9n3/2216-0973-cuid-9-3-2387.pdf. Acesso em 06 de janeiro de 2020.

PASSOS, D. R. et. al. Comportamento alimentar infantil: comparação entre crianças sem e com excesso de peso em uma escola do município de Pelotas, RS. Revista Paulista de Pediatria, São Paulo, v.33, p. 42-49, 2015. Disponível em: http://www.scielo.br/pdf/rpp/v33n1/pt_0103-0582-rpp-33-01-00042.pdf. Acesso em 06 de janeiro de 2020.

PEREIRA, C. S. N; BARROS, D. F e HEMAIS. A escola como ambiente obesogênico: as influências sobre a alimentação infantil, a partir da vulnerabilidade do consumidor. Diálogo com a Economia Criativa, Rio de Janeiro, v. 4, n. 10, p. 20-33, jan./abr. 2019. Disponível em: http://revistasrio.espm.br/index.php/revistadcec-rj/article/view/180/pdf. . Acesso em 06 de janeiro de 2020.

SILVA, C.M; TEIXEIRA, T. Comportamento alimentar infantil e atitudes parentais face à alimentação das crianças. Actas do $12^{\circ}$ Congresso Nacional de Psicologia da Saúde. Janeiro de 2018, Lisboa: ISPA - Instituto Universitário. Disponível em: http://repositorio.ispa.pt/bitstream/10400.12/6417/1/12CongNacSaude_231.pdf. Acesso em: Acesso em 06 de janeiro de 2020.

SILVA, R.C.R. DA, MALINA, R. M. Nível de atividade física em adolescentes no município de Niterói, Rio de Janeiro, Brasil. Caderno de Saúde Pública. Rio de Janeiro, v. 16, n. 4, out-dez, 2000. Disponível em https://www.scielosp.org/pdf/csp/2000.v16n4/1091-1097/pt. Acesso em 06 de janeiro de 2020 .

SOARES, A. C.O. et. al Marketing Nutricional e Direito à Saúde. Curitiba : Appris, 1 ed, 2018.

WHO/OMS. Organização Mundial da Saúde. Obesidade e excesso de peso. 2016. Disponível em: https://www.who.int/en/news-room/fact-sheets/detail/obesity-and- overweight . Acesso em 06 de janeiro de 2020. 